\title{
Multiwavelength Photometric Observations of Northern Carbon Stars
}

\author{
ANATOLY S. MIROSHNICHENKO ${ }^{1}$, \\ KENESKEN S. KURATOV ${ }^{2}$, \\ ŽELJKO IVEZIĆ ${ }^{3}$, and MOSHE ELITZUR ${ }^{3}$
}

${ }^{1}$ Central Astronomical (Pulkovo) Observatory, St. Petersburg, Russia

${ }^{2}$ Fesenkov Astrophysical Institute, Almaty, Kazakhstan

${ }^{3}$ University of Kentucky, Lexington KY, U.S.A.

We discuss and justify the importance of simultaneous, multiwavelength observations of late-type stars. Such observations have a crucial role in constraining theoretical models of these objects and determining properties of the circumstellar medium. Performed in the visual and near-infrared wavelength range, they are also a valuable complement to the high-quality Low Resolution Spectra obtained by the Infrared Astronomical Satellite.

Here we report first results of our UBVRIJHK simultaneous observations of four northern carbon stars (S Cep, VY UMa, RY Dra, and Y CVn). The observations were performed at the Tien Shan Astronomical Observatory (Kazakhstan) in August 1995 and January 1996. Simultaneous multiwavelength photometry for S Cep was obtained for the first time. Comparison with previous observations for RY Dra, VY UMa, and Y CVn shows that their color indices have changed, and that RY Dra and Y CVn were observed at fainter brightness levels than previously. The monitoring of these stars will be continued and the sample size will be extended. 